PROCEEDINGS OF THE

AMERICAN MATHEMATICAL SOCIETY

Volume 00, Number 0, Pages 000-000

S $0002-9939(\mathrm{XX}) 0000-0$

\section{A PREDATOR-PREY SYSTEM WITH HOLLING-TYPE FUNCTIONAL RESPONSE}

\author{
NABIL BEROUAL AND TEWFIK SARI
}

(Communicated by Wenxian Shen)

\begin{abstract}
We consider the Gause-type predator-prey system with a large class of growth and response functions, in the case where the response function is not smooth at the origin. We discuss the conditions under which this system has exactly one stable limit cycle or has a positive stable equilibrium point and we describe the basin of attraction of the stable limit cycle and the stable equilibrium point, respectively. Our results correct previous results of the existing literature obtained for the Holling response function $x^{p} /\left(a+x^{p}\right)$, in the case where $0<p<1$.
\end{abstract}

$7 \quad$ where ${ }^{\circ}=d / d t ; x$ and $y$ represent the prey population and the predator population, B respectively; $\mu$ and $D$ are positive parameters. Here $x f(x)$ is the growth function of 9 the prey species in the absence of the predators, and $g(x)$ represents the functional 10 response of predators to the growth of prey. For the background on this model, see 11 Freedman [4].

In mathematical models for biology, the focus is on the global behavior of the system in the positive cone of the phase plane. The global behavior of the system is typically determined by the existence of a positive global attractor, either an equilibrium or a limit cycle. For this reason, the existence of a positive equilibrium of (1.1), its global asymptotic stability, the existence of a limit cycle (in case where the positive equilibrium is unstable), the uniqueness of the limit cycle, its global orbital attractivity, have attracted much interest in recent years, see $[6,7,8,9,11$, $12,13,14,15]$ and the references therein.

The functions $f$ and $g$ in (1.1) are assumed to satisfy the following hypotheses.

(H1) $f$ is smooth for $x \geq 0$ and there exists $k>0$ such that $f(k)=0$ and $(x-k) f(x)<0$ for $x \geq 0$ and $x \neq k$.

(H2) $g$ is continuous for $x \geq 0, g(0)=0$ and $\mu g(k)>D$. It is smooth for $x>0$ and $g^{\prime}(x)>0$ for $x>0$.

Received by the editors November 4, 2019.

2010 Mathematics Subject Classification. Primary 34A12, 34C05, 34D23, Secondary 70K05, 92D25. 
In (H1) the value $k$ is interpreted as the carrying capacity of the prey species in the absence of the predators. The system (1.1) admits two boundary equilibrium points, $E_{0}=(0,0)$ and $E_{1}=(k, 0)$. It follows from (H2) that there is a unique $x^{*}$ such that $0<x^{*}<k$ and $g\left(x^{*}\right)=D / \mu$. The predator isocline in the positive cone, i.e., the curve determined by the equation $\mu g(x)-D=0$, is a vertical line $x=x^{*}$. Meanwhile, the prey isocline is the graph of the function $y=\varphi(x)$ where

$$
\varphi(x)=\frac{x f(x)}{g(x)} .
$$

It is easy to see that $\varphi(k)=0$ and $\varphi(x)>0$ for $0<x<k$. The value of $\varphi(0)$ depends on additional properties on $g$ near the origin. The intersection point of the isoclines, $E^{*}=\left(x^{*}, y^{*}\right)$ with $y^{*}=\varphi\left(x^{*}\right)$, is the only positive equilibrium point of system (1.1).

Since $g$ is not assumed to be smooth at $0, E_{0}$ is not a hyperbolic equilibrium. Using linear stability analysis (Jacobian) it is easy to see that $E_{1}$ is always a saddle point, while $E^{*}$ can be a stable or unstable focus or node depending on the sign of $\varphi^{\prime}\left(x^{*}\right)$, where $\varphi$ is defined by (1.2). For $\varphi^{\prime}\left(x^{*}\right)<0, E^{*}$ is an attractor. For $\varphi^{\prime}\left(x^{*}\right)>0, E^{*}$ is a repeller.

A prototype of $f(x)$ and $g(x)$ in (1.1) is given by the logistic growth and the Holling functional response, see [12, 13],

$$
f(x)=r\left(1-\frac{x}{k}\right), \quad g(x)=\frac{x^{p}}{a+x^{p}},
$$

where $r, k, a$ and $p$ are positive parameters ( $p$ is not always an integer). In this case, if $0<p<1$, the function $x \mapsto g(x)$ is not smooth at $x=0$. Using numerical simulations, it was observed in [2] that, if $0<p<1$, some results of [12, 13] on the existence of a limit cycle or on the global stability of the positive equilibrium, are not true. We show in this paper why the classical arguments of dynamical systems do not apply, and why the results on existence and uniqueness of limit cycle, together with the result on global attractivity of the positive equilibrium or the limit cycle obtained in $[12,13]$ are not true when $0<p<1$.

Liu [9] considered the system (1.1). This author did not required the smoothness of $g(x)$ at $x=0$, because (see the lines following Hypothesis (H2) in [9]), in some models it may happen that

$$
g^{\prime}\left(0^{+}\right):=\lim _{x \rightarrow 0^{+}} \frac{g(x)}{x}=+\infty .
$$

For instance, for Holling functions (1.3) with $0<p<1$, (1.4) holds.

The purpose of this paper is to consider the case where $g(x)$ is not smooth at $x=0$ and to study the global asymptotic stability of the positive equilibrium point (when it is locally exponentially stable), and also to study the existence of limit cycles (when the positive equilibrium is unstable) and the orbital asymptotic stability, and hence uniqueness, of the limit cycle (when the limit cycle exists).

The paper is organized as follows. In the next section we give a condition on the response function $g$, which is satisfied by the Holling function (1.3), with $0<$ $p<1$, such that all points of the positive $y$-axis are points of non-uniqueness of the solutions of (1.1), that is to say the Cauchy problem (1.1) and the conditions $x(0)=0, y(0)>0$ has not only the trivial solution $x(t) \equiv 0$, but also has solutions satisfying $x(t)>0$ for some $t<0$. In Section 3 we discuss the global results on (1.1) and we describe the basin of attraction of the limit cycle, if it exists, and the 
basin of attraction of the positive equilibrium point, when it is a local attractor. In Section 4 we apply our results to the system (1.1) where $f$ and $g$ are given by (1.3) and we correct some of the results in the existing literature which asserted that the system has a unique limit cycle (which is hence globally attracting), if and only if the positive equilibrium is a repeller, and that the positive equilibrium is globally attracting if and only if it is a local attractor. Some complements and technical proofs are given in the appendix.

\section{Non Uniqueness of SOlutions}

The function $g(x)$ is assumed to satisfy the following hypothesis.

(H3) $g(x)$ is not smooth for $x=0,(1.4)$ holds and the integral $\int_{\varepsilon}^{\alpha} \frac{d x}{g(x)}$ converges as $\varepsilon \rightarrow 0$, where $\alpha>0$ is fixed.

It follows from (1.4) that $\varphi(0)=0$. The condition on the convergence of the integral in (H3) is a technical condition that will be useful for the proofs presented in this paper. For instance, for Holling functions (1.3) with $0<p<1$, (H2) and (H3) hold. However, it should be noticed that (1.4) does not imply that the improper integral $\int_{\varepsilon}^{\alpha} \frac{d x}{g(x)}$ converges. For instance if function $g$ is equivalent to $-x \ln (x)$ near 0 , then (1.4) hods and the improper integral does not converge.

Let $x_{0}$ be such that $0<x_{0}<x^{*}$. Let $\left(X\left(t, x_{0}\right), Y\left(t, x_{0}\right)\right)$ be the solution of (1.1) with initial condition $\left(x_{0}, \varphi\left(x_{0}\right)\right)$. As long as $x^{*}>x>0$ and $y>\varphi(x)$, the solution $\left(X\left(t, x_{0}\right), Y\left(t, x_{0}\right)\right)$ can be parametrized by $x$, that is to say its orbit is the graph of a function of $x$ :

$$
y=\eta\left(x, x_{0}\right), \text { for } x_{0} \leq x \leq x^{*} .
$$

Our aim is to show that the limit of these orbits (2.1) exists when $x_{0} \rightarrow 0$ and is a positive orbit. If the equilibrium point $E_{0}$ was hyperbolic, then the limit of orbits (2.1) is simply the $y$-axis, which is the stable separatrix of $E_{0}$. However, under hypothesis $(\mathrm{H} 3)$ all points $\left(0, y_{0}\right)$, with $y_{0}>0$ are non uniqueness points and some solutions with initial conditions $x(0)>0, y(0)>0$ can reach in finite time the $y$-axis at some point $\left(0, y_{0}\right)$ after with $y$ decreases to 0 . Therefore the orbits (2.1) are bounded above, so that their limit exists and is a positive orbit.

More precisely, we consider the initial value problem

$$
\begin{array}{ll}
\dot{x}=x f(x)-y g(x), & x(0)=0, \\
\dot{y}=y(\mu g(x)-D), & y(0)=y_{0} .
\end{array}
$$

Besides the trivial solution $\left(x(t)=0, y(t)=y_{0} e^{-D t}\right)$, the initial value problem (2.2) admits nontrivial positive solutions $\left(x_{1}(t), y_{1}(t)\right)$, where $x_{1}(t)$ is not identically 0 , which tends also to $E_{0}$ as $t \rightarrow+\infty$, as shown in the following result.

Theorem 2.1. For any $y_{0}>0,\left(0, y_{0}\right)$ is a non uniqueness point for (2.2). The system admits a maximal solution $\left(X_{1}\left(t, y_{0}\right), Y_{1}\left(t, y_{0}\right)\right)$ satisfying

(1) $X_{1}\left(t, y_{0}\right)>0$ and $Y_{1}\left(t, y_{0}\right)>0$ for $t<0$.

(2) $X_{1}\left(t, y_{0}\right)=0$ and $Y_{1}\left(t, y_{0}\right)=y_{0} e^{-D t}$ for $t \geq 0$.

Proof. The concept of maximal solution used in this theorem should not be confused with the usual concept of maximal solution, defined on a maximal interval of definition. Here the solution is said to be maximal in the sense that it is the solution $\left(X_{1}(t), Y_{1}(t)\right)$ of the initial value problem $(2.2)$, which is non trivial (i.e. 
$X_{1}(t)>0$ ), on the maximal possible interval, that is to say for all $t<0$. The details of the proof is given in Appendix B.

As long as $0<x<x^{*}$ and $y>\varphi(x)$, the solution $\left(X_{1}\left(t, y_{0}\right), Y_{1}\left(t, y_{0}\right)\right)$ can be parametrized by $x$, that is to say, its orbit is the graph of a function of $x$ :

Hence, we have the following inequalities, see Fig. 1(a):

$$
\varphi(x)<\eta\left(x, x_{0}\right)<\eta_{1}\left(x, y_{0}\right) \text {, for } x_{0}>0, y_{0}>0 \text { and } x_{0} \leq x \leq x^{*} .
$$

Therefore the following limits

$$
\begin{aligned}
& w(x)=\lim _{x_{0} \rightarrow 0} \eta\left(x, x_{0}\right)=\sup _{0<x_{0}<x^{*}} \eta\left(x, x_{0}\right), \\
& w_{1}(x)=\lim _{y_{0} \rightarrow 0} \eta_{1}\left(x, y_{0}\right)=\inf _{y_{0}>0} \eta_{1}\left(x, y_{0}\right),
\end{aligned}
$$

exit and satisfy $\varphi(x)<w(x) \leq w_{1}(x)$, for $0<x<x^{*}$, see Fig. 1(a). Since the graphs of functions $y=w(x)$ and $y=w_{1}(x)$ are limits, when $x_{0} \rightarrow 0$ and $y_{0} \rightarrow 0$ respectively, of orbits of (1.1), they themselves are orbits of (1.1). We denote by $W^{s}\left(E_{0}\right)$ and $W_{1}^{s}\left(E_{0}\right)$ the global orbits corresponding to the local orbits $y=w(x)$ and $y=w_{1}(x)$ respectively, see Fig. 1(b).

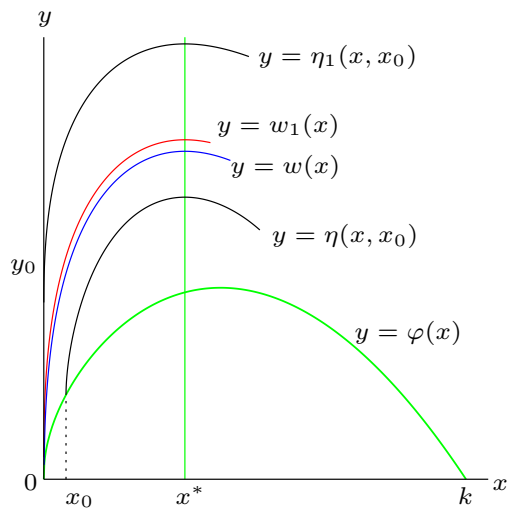

(a)

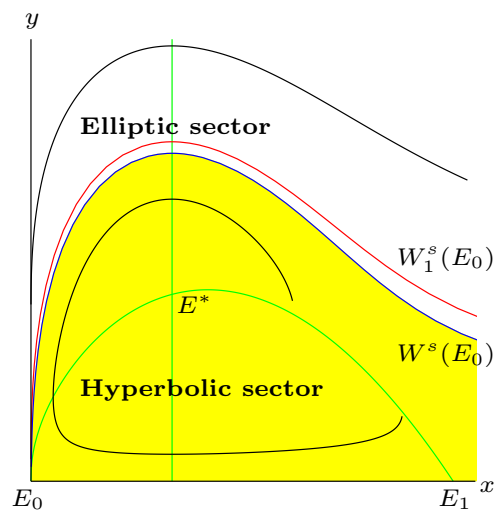

(b)

Figure 1. (a): The definitions of the local orbits $y=w(x)$ (colored in blue) and $y=w_{1}(x)$ (colored in red). (b): The global corresponding orbits $W^{s}\left(E_{0}\right)$ (colored in blue) and $W_{1}^{s}\left(E_{0}\right)$ (colored in red) and the elliptic sector, above $W^{s}\left(E_{0}\right)$, and hyperbolic sector, below $W^{s}\left(E_{0}\right)$ (colored in yellow), of $E_{0}$.

Remark 2.2. Although the origin $E_{0}$ is not a hyperbolic singular point, we use the notations $W^{s}\left(E_{0}\right)$ and $W_{1}^{s}\left(E_{0}\right)$ since the solutions corresponding to these orbits tend to $E_{0}$ as $t \rightarrow+\infty$. The description of the behavior of the system near $E_{0}$ is as follows: The region between $W^{s}\left(E_{0}\right)$ and the $x$-axis is a hyperbolic sector and the region between $W^{s}\left(E_{0}\right)$ and the $y$-axis is an elliptic sector, see Fig. 1(b). Orbits who are between $W^{s}\left(E_{0}\right)$ and $W_{1}^{s}\left(E_{0}\right)$ tend toward $E_{0}$ when $t \rightarrow+\infty$ and those who are between the $y$ axis and $W_{1}^{s}\left(E_{0}\right)$ reach the $y$ axis in finite time and then, go toward the origin when $t \rightarrow+\infty$. 


\section{Global Behaviour}

Assume that $E^{*}$ is a repeller, that is to say $\varphi^{\prime}\left(x^{*}\right)>0$. If $g$ were smooth at 0 , then the system would necessarily have a limit cycle surrounding $E^{*}$. The existence of a limit cycle results from the Poincaré-Bendixson Theorem and the fact that $E_{0}$ is a saddle point, see Appendix A. Is this limit cycle globally orbitally asymptotically stable (GOAS) ? If the system has at most one cycle, then this cycle is necessarily GOAS.

Assume that $E^{*}$ is an attractor, that is to say $\varphi^{\prime}\left(x^{*}\right)<0$. Is $E^{*}$ globally asymptotically stable (GAS) ? In the case where $g$ is smooth at 0 , if a solution with positive initial condition does not converge toward $E^{*}$, then, from the PoincaréBendixson Theorem and the fact that $E_{0}$ is a saddle point, it must converge toward a limit cycle, see Appendix A. If the system cannot have a cycle, then $E^{*}$ is necessarily GAS.

In both cases the argument that the system has a limit cycle that must surround $E^{*}$ uses the fact that $E_{0}$ is a saddle point, which does not hold when $g$ is not smooth at 0 , as shown in the preceding section, see Remark 2.2. In the case where hypothesis (H3) holds, $E_{0}$ is not a saddle point and the Poincaré-Bendixson theorem is inapplicable. In this case, the description of the global behavior of the system is obtained by considering the relative position of the orbit $W^{s}\left(E_{0}\right)$ with respect to the unstable separatrix $W^{u}\left(E_{1}\right)$ of the saddle point $E_{1}$.

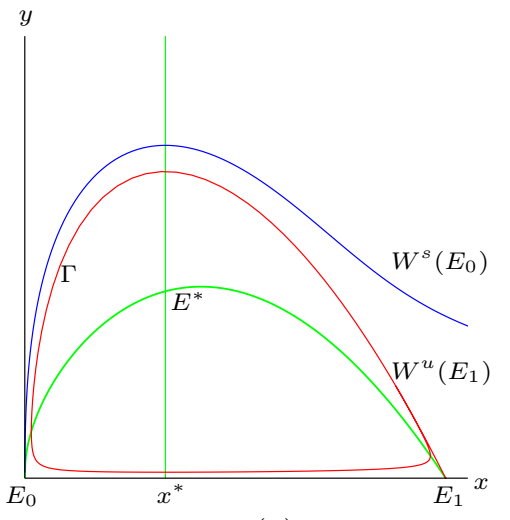

(a)

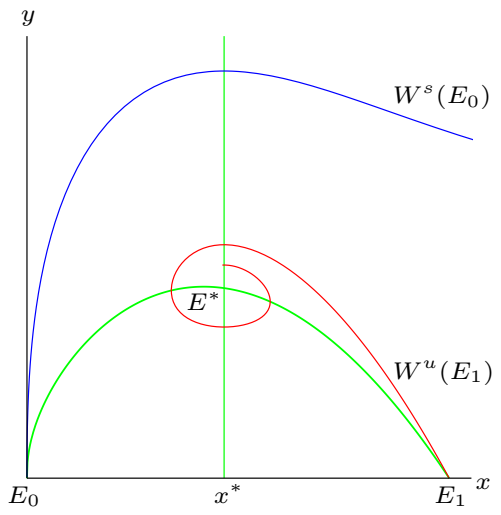

(b)

Figure 2. $W^{s}\left(E_{0}\right)$ (in blue) is above $W^{u}\left(E_{1}\right)$ (in red). The isoclines $\dot{x}=0$ and $\dot{y}=0$ are represented in green. (a): $E^{*}$ is a repeller; (b): $E^{*}$ is an attractor.

3.1. The case where $W^{s}\left(E_{0}\right)$ is above $W^{u}\left(E_{1}\right)$. We have the following result

Proposition 3.1. Assume that $W^{s}\left(E_{0}\right)$ is above $W^{u}\left(E_{1}\right)$. If $E^{*}$ is a repeller, then it is surrounded by at least one limit cycle. If the system can have at most one cycle, then $E^{*}$ is surrounded by a unique limit cycle which is orbitally asymptotically stable. This limit cycle is not GOAS, even if it is unique. If $E^{*}$ is an attractor and if the system has no cycles, then all orbits below $W^{s}\left(E_{0}\right)$ converge towards $E^{*} . E^{*}$ is not GAS, even if it is not surrounded by any unstable limit cycle. 


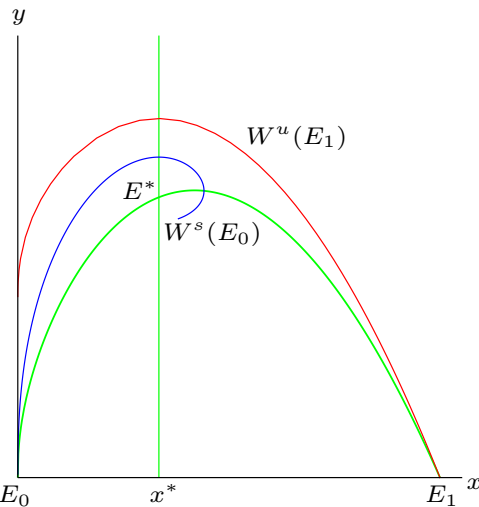

(a) $2(\mathrm{~b})$.

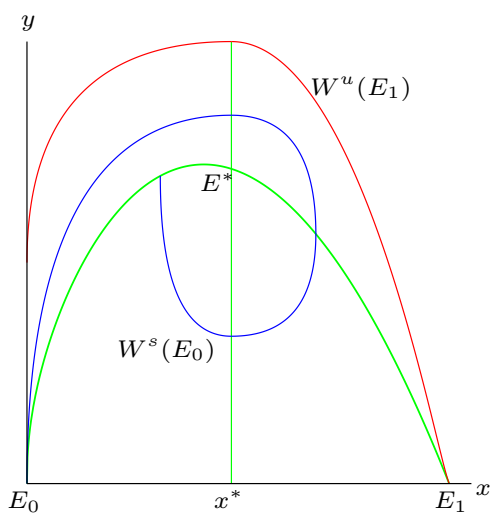

(b)

Figure 3. $W^{s}\left(E_{0}\right)$ (in blue) is below $W^{u}\left(E_{1}\right)$ (in red). The isoclines $\dot{x}=0$ and $\dot{y}=0$ are represented in green. (a): $E^{*}$ is a repeller; (b): $E^{*}$ is an attractor.

Proof. Assume that $E^{*}$ is a repeller. The orbit $W^{u}\left(E_{1}\right)$ lies in the hyperbolic sector. Therefore, its $\omega$-limit set is a limit cycle $\Gamma$ surrounding $E^{*}$. This limit cycle is attracting at least on its exterior, see Fig. 2(a). Since $E^{*}$ is repelling, it is surrounded also by a limit cycle $\Gamma_{1}$ which is attracting at least on its interior. If the system can have at most one cycle then $\Gamma_{1}=\Gamma$. Therefore a limit cycle exists but it is not GOAS: all orbits above $W^{s}\left(E_{0}\right)$ converge toward $E_{0}$ and all positive orbits below $W^{s}\left(E_{0}\right)$, excepted for $E^{*}$, converge toward the limit cycle $\Gamma$.

Assume that $E^{*}$ is an attractor. The orbit $W^{u}\left(E_{1}\right)$ lies in the hyperbolic sector. Therefore, its $\omega$-limit set, if it does not contain $E^{*}$, is necessarily a limit cycle surrounding $E^{*}$. If the system cannot have a cycle then the limit set of $W^{u}\left(E_{1}\right)$ is equal to $E^{*}$. Therefore $E^{*}$ is not GAS since: all orbits above $W^{s}\left(E_{0}\right)$ converge toward $E_{0}$ and all positive orbits below $W^{s}\left(E_{0}\right)$ converge toward $E^{*}$, see Fig.

\subsection{The case where $W^{s}\left(E_{0}\right)$ is below $W^{u}\left(E_{1}\right)$. We have the following result}

Proposition 3.2. Assume that $W^{s}\left(E_{0}\right)$ is below $W^{u}\left(E_{1}\right)$. If $E^{*}$ is a repeller and assuming that the system can have at most one cycle and, if it exists, this cycle cannot be semi-stable (i.e stable on one side and unstable in another side), then all positive orbits, excepted for $E^{*}$, converge toward $E_{0}$. If $E^{*}$ is an attractor then it is surrounded by an unstable limit cycle.

Proof. Assume that $E^{*}$ is a repeller. The orbit $W^{s}\left(E_{0}\right)$ lies in the hyperbolic sector. Therefore, its $\alpha$-limit set, if it does not contain $E^{*}$, is necessarily a limit cycle $\Gamma$ surrounding $E^{*}$. This limit cycle is repelling on its exterior. Since $E^{*}$ is repelling, it is surrounded also by a limit cycle $\Gamma_{1}$ which is attracting on its interior. Since the system can have at most one cycle, $\Gamma_{1}=\Gamma$. Therefore $\Gamma$ is attracting on one side and repelling on the other side. Since this situation is impossible, the $\alpha$-limit set of $W^{s}\left(E_{0}\right)$ is equal to $E^{*}$. Therefore no limit cycle can exist and all positive solutions, excepted for $E^{*}$, converge toward $E_{0}$. 

by $E_{0}$.

Assume that $E^{*}$ is an attractor. The orbit $W^{s}\left(E_{0}\right)$ lies in the hyperbolic sector. Therefore, its $\alpha$-limit set is necessarily a limit cycle $\Gamma$ surrounding $E^{*}$. This limit cycle is repelling on its exterior.

The first case of the proposition ( $E^{*}$ is a repeller) is depicted in Fig.3(a). This case presents an unstable positive equilibrium, which is not surrounded by a cycle. The second case of the proposition ( $E^{*}$ is an attractor) is depicted in Fig. 3(b). If the system cannot have a cycle then the situation where $E^{*}$ is an attractor and in the same time $W^{s}\left(E_{0}\right)$ is below $W^{u}\left(E_{1}\right)$ cannot hold.

\section{Applications}

The purpose of this section is to clarify the necessary and sufficient conditions for the existence and uniqueness of limit cycles of the predator-prey system (1.1), where $f$ and $g$ are given by (1.3), with $0<p<1$. The system is written

$$
\begin{aligned}
& \dot{x}=r x\left(1-\frac{x}{k}\right)-\frac{x^{p} y}{a+x^{p}}, \\
& \dot{y}=y\left(\frac{\mu x^{p}}{a+x^{p}}-D\right) .
\end{aligned}
$$

The condition $\mu g(k)>D$ in (H2) is equivalent to $k>x^{*}$, where $x^{*}$ is defined by $g\left(x^{*}\right)=D / \mu$, that is to say

$$
\mu>D, \quad k>x^{*}:=\left(\frac{a D}{\mu-D}\right)^{\frac{1}{p}} .
$$

Hence functions $f$ and $g$ satisfy the hypotheses (H1), (H2) and (H3) if and only if $0<p<1$ and (4.2) is satisfied. Therefore the results in Propositions 3.1 and 3.2, have the corollary that if $E^{*}$ is a repellor, the system does not always have a limit cycle, and if $E^{*}$ is a attractor, it is not GAS.

4.1. Existence of a limit cycle. Sugie, Kohno and Miyazaki have stated (see Theorem 2.1 in [13]) that if

$$
(p D-(p-1) \mu) k>(p D-(p-2) \mu) x^{*},
$$

then the system (4.1) has a unique limit cycle. Owing the the non uniqueness of the solution, this claim is not true. We have the following result:

Proposition 4.1. Assume that (4.2) and (4.3) hold. Then, if $W^{s}\left(E_{0}\right)$ is above $W^{u}\left(E_{1}\right)$, the system (4.1) has a unique limit cycle whose basin of attraction is the positive region of the plane located below $W^{s}\left(E_{0}\right)$. If $W^{s}\left(E_{0}\right)$ is below $W^{u}\left(E_{1}\right)$, the system (4.1) has no limit cycle and all positive solutions, excepted for $E^{*}$ converge toward $E_{0}$.

Proof. One can check that the condition (4.3) is equivalent to $\varphi^{\prime}\left(x^{*}\right)>0$. Therefore $E^{*}$ is a repellor and it can be surrounded by a limit cycle. However, this limit cycle is not GOAS since it cannot attract the orbits above $W^{s}\left(E_{0}\right)$. Actually, if it exists, the limit cycle attracts all the orbits below $W^{s}\left(E_{0}\right)$. Using the result of [13] that, in this case, the system can have at most one limit cycle, we obtain as a corollary of Proposition 3.1 that if $W^{s}\left(E_{0}\right)$ is above $W^{u}\left(E_{1}\right)$, then the system has a unique limit cycle. On the other hand, using Proposition 3.2 we obtain that if $W^{s}\left(E_{0}\right)$ is below $W^{u}\left(E_{1}\right)$, then the system has no cycle and all positive solutions are attracted 


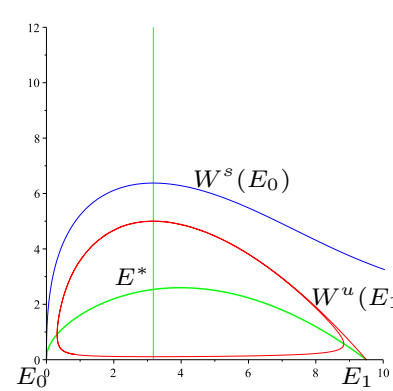

(a)

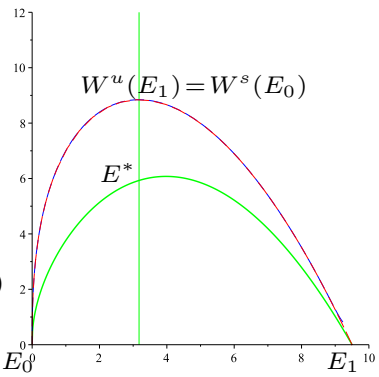

(b)

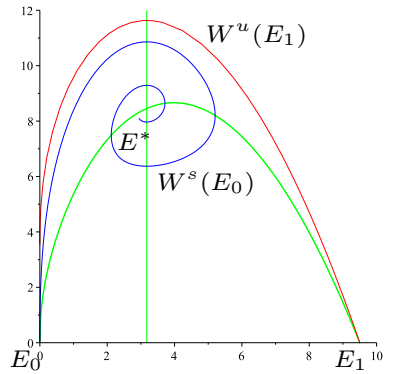

(c)

Figure 4. The relative positions of $W^{s}\left(E_{0}\right)$ and $W^{u}\left(E_{1}\right)$ for (4.1) when the parameter values are $k=9.5, a=2, p=0.6, \mu=2$, $D=1$ and (a): $r=0.6,(\mathrm{~b})$ : The heterocline bifurcation $W^{s}\left(E_{0}\right)=$ $W^{u}\left(E_{1}\right)$ holds for $1.4026<r<1.4027$, (c): $r=2$.

These results are illustrated in Fig. 4. The numerical simulations show that for the given values of the parameters, there exists a bifurcation value $r_{0}$ for which the heterocline bifurcation $W^{s}\left(E_{0}\right)=W^{u}\left(E_{1}\right)$ occurs: If $r<r_{0}$ then $W^{s}\left(E_{0}\right)$ is above $W^{u}\left(E_{1}\right)$ and the system has a unique limit cycle which is not GOAS; If $r>r_{0}$ then $W^{s}\left(E_{0}\right)$ is below $W^{u}\left(E_{1}\right)$ and the system has no limit cycle, and the positive solutions are attracted by the origin.

4.2. Global asymptotic stability of $E^{*}$. Sugie and Katayama have stated (see Theorem 1.1 in [12]) that the positive equilibrium $E^{*}$ is GAS if and only if

$$
(p D-(p-1) \mu) k \leq(p D-(p-2) \mu) x^{*} .
$$

Owing the the non uniqueness of the solution, this claim is not true. We have the following result:

Proposition 4.2. Assume that (4.2) and (4.4) hold. Then $W^{s}\left(E_{0}\right)$ is above $W^{u}\left(E_{1}\right)$ and the basin of attraction of $E^{*}$ is the positive region of the plane located below $W^{s}\left(E_{0}\right)$. Therefore $E^{*}$ is not $G A S$.

Proof. One can check that the condition (4.4) is equivalent to $\varphi^{\prime}\left(x^{*}\right) \leq 0$. Therefore $E^{*}$ is an attractor. However, it is not GAS since it cannot attract the orbits above $W^{s}\left(E_{0}\right)$. Actually, it attracts all the orbits below $W^{s}\left(E_{0}\right)$. Using the result of $[9,12]$ that, in this case the system has no cycle, we obtain as a corollary of Proposition 3.2 that $W^{s}\left(E_{0}\right)$ is always above $W^{u}\left(E_{1}\right)$, that is to say, the situation depicted in Fig. 3 never occurs. Therefore, using Propositions 3.1 we obtain that $E^{*}$ attracts all the orbits below $W^{s}\left(E_{0}\right)$.

These results are illustrated in Fig. 5, where it is shown that $E^{*}$ is never GAS.

\section{Discussion}

We have shown in this work that the differentiability of $g$ at $x=0$ could drastically affect the dynamics of (1.1). For instance, when $0<p<1$, and $E^{*}$ is a repeller, the system (4.1) does not necessarily have a limit cycle. When $W^{s}\left(E_{0}\right)$ is above $W^{u}\left(E_{1}\right)$, the limit cycle exists. However, it is not GOAS. All solutions with initial conditions above $W^{s}\left(E_{0}\right)$ reach the $y$-axis in finite time. All solutions with initial conditions below $W^{s}\left(E_{0}\right)$ excepted the equilibrium point $E^{*}$ converge to the 


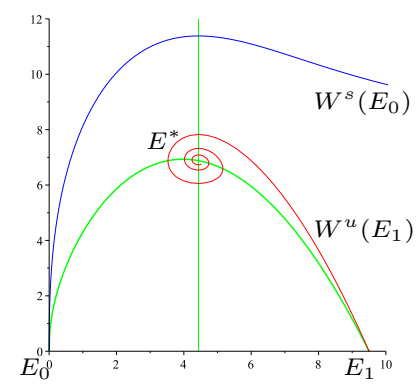

Figure 5. When $E^{*}$ is an attractor, $W^{s}\left(E_{0}\right)$ is always above $W^{u}\left(E_{1}\right)$. The parameter values are the same as in Fig. 4, excepted that $D=1.1$ and $r=1.6$.

limit cycle. When $W^{s}\left(E_{0}\right)$ is below $W^{u}\left(E_{1}\right)$, the limit cycle does not exist. All solutions, excepted the equilibrium point $E^{*}$, reach the $y$-axis in finite time.

An heterocline bifurcation occurs when $W^{s}\left(E_{0}\right)=W^{u}\left(E_{1}\right)$ giving rise to a polycycle: all solutions with initial conditions outside the poly-cycle reach the $y$-axis in finite time. All solution with initial conditions inside the poly-cycle excepted the equilibrium $E^{*}$ converge to the poly-cycle.

On the other hand, when $0<p<1$ and $E^{*}$ is an attractor, it is not GAS. In this case $W^{s}\left(E_{0}\right)$ is always above $W^{u}\left(E_{1}\right)$, and the basin of attraction of $E^{*}$ is the positive region below $W^{s}\left(E_{0}\right)$.

Our findings clarify some misunderstandings on the behaviour of this prey predator model that appeared in the existing literature $[12,13]$. It is not true that when $0<p<1$ the system admits a limit cycle as long as its positive equilibrium $E^{*}$ is a repeller, nor that this limit cycle is globally attracting whenever it exists, nor that $E^{*}$ is GAS, whenever it is an attractor.

In [12], the authors consider that the function $g(x)=\frac{x^{p}}{a+x^{p}}$ in (4.1) is of Holing type II if $p \leq 1$ and of Holing type III if $p>1$. Actually the functional response belongs to Holling type III if $p>1$, since its has an inflexion point and the functional response curve is sigmoid [5]. When $0<p \leq 1$, the function $g$ is strictly increasing, bounded and convex, although the case $0<p<1$ deserves special interest because of the singularity of $g$ in 0 . From a biological and ecological point of view, the functional response $g$ with $0<p<1$, defines a class of prey and predator populations characterized by predators with a very high attack rate for very small densities prey and a need for a large amount of prey to reach satiety. This response function has the property that the prey can go extinct in finite time, which never happens when $p \geq 1$.

\section{Appendix A. Existence of Limit CyCles When $g$ is Smooth at 0}

Most of the classical works on (1.1) or (4.1) have employed the transformation that converts the system into an equivalent generalized Liénard system. This method requires that $f(x)$ and $g(x)$ are sufficiently smooth so that existence, uniqueness, and continuation for all positive $t$ are satisfied for initial-value problems. For instance, the proof of the existence of a cycle, when $g$ is smooth at 0 , and the positive equilibrium is a repeller, needs the Poincaré-Bendixson Theorem 
and the fact that $E_{0}$ is hyperbolic. However, since this proof is standard, it is often omitted in the existing literature by authors who attempted to extend the results to functions $g$ which are not smooth at 0 . This is precisely the point where these proofs are not correct.

For the convenience of the reader, we give here the details on the proof of the existence of a limit cycle, when the positive equilibrium point is a repeller and the function $g$ is smooth at 0 . The hypothesis of hyperbolicity of $E_{0}$ is crucial to applying the so-called Butler-MacGehee Theorem which we recall here and whose proof is given in [10].

Theorem A.1. (Butler-McGehee). If an omega (or alpha)-limit set $\omega$ contains a hyperbolic equilibrium point $a$, but it is not equal to $\{a\}$, then $\omega$ contains a point $b \neq a$ of the stable manifold $W^{s}(a)$, as well as a point $c \neq a$ of the unstable manifold $W^{u}(a)$.

We have the following result

Theorem A.2. Assume that (H1) and (H2) hold and in addition $g$ is smooth at 0 . If $E^{*}$ is a repeller, then (1.1) has a limit cycle that contains $E^{*}$ in its interior.

Proof. It is known that under the hypotheses (H1) and (H2), all solutions of (1.1) are positively bounded. Therefore the omega-limit set $\omega$ of a positive trajectory is nonempty, invariant and compact. If $g$ is smooth at $x=0$ then $E_{0}$ is hyperbolic (a saddle point).

The limit set $\omega$ does not contain the saddle points $E_{0}$ and $E_{1}$. Indeed, if $\omega$ contains $E_{0}$, and since it is different from $E_{0}$, because $E_{0}$ is the omega-limit set of only the trajectories lying in the $y$-axis, then by the Butler-MacGehee Theorem, it must contain a point $b=\left(0, y_{0}\right)$ with $y_{0}>0$ of $W^{s}\left(E_{0}\right)$ which is the positive $y$-axis. Since $\omega$ is invariant it must contain the whole trajectory of $b$, which is the positive $y$ axis. This contradicts the compactness of $\omega$.

In the same manner if $\omega$ contains $E_{1}$, and since it is different from $E_{1}$, because $E_{1}$ is the omega-limit set of only trajectories lying in the $x$-axis, then by the ButlerMacGehee Theorem, it must contain a point $b=\left(x_{0}, 0\right)$ with $x_{0}>0$ of $W^{s}\left(E_{1}\right)$ which is the positive $x$-axis. Two cases must be distinguished: If $x_{0}>k$ then $\omega$ contains the whole trajectory of $b$ which is unbounded. This contradicts the compactness of $\omega$; If $x_{0}<k$ then $\omega$ contains the whole trajectory of $b$ which is the segment $(0, k)$ of the $x$-axis. Since $\omega$ is closed, it must contain $[0, k]$, the closure of $(0, k)$. Therefore $\omega$ contains $E_{0}$ which is impossible as we have previously seen.

Assuming that $E^{*}$ is a repeller, then it cannot belong to the omega-limit set $\omega$. Therefore $\omega$ contains no equilibrium points. By the Poincaré-Bendixson Theorem, $\omega$ is a cycle. Since in the plane, a cycle must contain an equilibrium point in its interior, $\omega$ surrounds $E^{*}$.

If one have to our disposal the result that (1.1) admits at most one cycle, then the limit cycle whose existence was proved in the previous theorem is necessarily GOAS. Therefore, all solutions with positive initial conditions, excepted for $E^{*}$ converge toward this limit cycle. The solutions with initial conditions on the $y$-axis converge toward $E_{0}$, and the solutions with initial conditions on the positive $x$-axis converge toward $E_{1}$.

When $E^{*}$ is an attractor, we have the following result 
In the region

$$
D=\left\{(x, u): 0 \leq x<x^{*}, u>\ln \frac{\varphi(x)}{y_{0}}\right\},
$$

Theorem A.3. Assume that (H1) and (H2) hold and in addition $g$ is smooth at 0 . If $E^{*}$ is an attractor and (1.1) has no cycles then $E^{*}$ is GAS.

Proof. Assume that $E^{*}$ is an attractor. If a solution with positive initial condition does not converge toward $E^{*}$, then its omega-limit set $\omega$ cannot contain $E^{*}$. By the Butler-McGehee Theorem, it cannot contain $E_{0}$ nor $E_{1}$ as shown in the proof of the previous theorem. Therefore $\omega$ contain no equilibrium points. From the Poincaré-Bendixson Theorem, $\omega$ is a limit cycle, which contradicts the hypothesis that (1.1) has no cycles. Therefore $\omega=\left\{E^{*}\right\}$, which proves that $E^{*}$ is GAS.

In the case where $g(x)$ is not smooth at $x=0, E_{0}$ is not a hyperbolic saddle point and the arguments used in the proofs of Theorem A.2 and Theorem A.3 do not apply. For instance, in his proof of Theorem 1 in [9], the author claimed that $(0,0)$ is a saddle, which is not true when $g(x)$ is not smooth at $x=0$. Therefore, the system does not have necessarily a cycle when $\left(x^{*}, y^{*}\right)$ is a repeller. Moreover, the claim of the author following the proof of his Theorem 1, that "local asymptotic stability of $\left(x^{*}, y^{*}\right)$ implies its global asymptotic stability" is not true. Indeed Fig. 5 provides a counterexample to this claim.

\section{Appendix B. Proof of Theorem 2.1}

The proof needs the following result, known as the Tamarkine's non uniqueness theorem (see [1], p. 98).

Theorem B.1. Let $F(u, x)$ be a continuous function on $S=[-a, a] \times[-b, b]$, with $a>0$ and $b>0$. Assume that for all $(u, x) \in S$

$$
\left|F(u, x)-F\left(u, x_{0}(u)\right)\right| \geq G\left(\left|x-x_{0}(u)\right|\right),
$$

where $u \mapsto x_{0}(u)$ is a solution of the initial value problem

$$
\frac{d x}{d u}=F(u, x), \quad x(0)=0 .
$$

Assume that $z \mapsto G(z)$ is an increasing function for $z \geq 0, G(0)=0$ and the integral $\int_{\varepsilon}^{\alpha} \frac{d z}{G(z)},(\alpha>\varepsilon)$ converges as $\varepsilon \rightarrow 0$. Then, the initial value problem (B.1) has at least two solutions in $[-a, a]$.

Proof. (of Theorem 2.1) The change of variables $y=y_{0} e^{u}$, transforms (2.2) into the system

where $\varphi(x)$ is defined by (1.2), we have $x f(x)-y_{0} e^{u} g(x)<0$ and $\mu g(x)-D<0$. Therefore (B.2) is equivalent to the scalar initial value problem

$$
\frac{d x}{d u}=F\left(u, x, y_{0}\right), \quad x(0)=0,
$$

with

$$
F\left(u, x, y_{0}\right)=\frac{x f(x)-y_{0} e^{u} g(x)}{\mu g(x)-D}
$$


where $y_{0}$ is considered as a parameter. Using $(1.2), F\left(u, x, y_{0}\right)$ can be written

$$
F\left(u, x, y_{0}\right)=g(x) \frac{y_{0} e^{u}-\varphi(x)}{D-\mu g(x)} .
$$

Since $g(0)=0, F\left(u, 0, y_{0}\right)=0$. Therefore $x_{0}(u)=0$ is a solution of (B.3). Actually the initial value problem (B.3) has also non trivial solutions.

If $u \geq 0$ then $y_{0} e^{u} \geq y_{0}$ and hence, for any $u \geq 0$ and $x \in\left[0, x^{*}\right)$, we have

$$
\left|F\left(u, x, y_{0}\right)-F\left(u, x_{0}(u), y_{0}\right)\right|=F\left(u, x, y_{0}\right)>G(x),
$$

where

Let us denote by

$$
G(z)=g(z) \frac{y_{0}-\varphi(z)}{D-\mu g(z)}
$$

$$
h(z)=\frac{y_{0}-\varphi(z)}{D-\mu g(z)} .
$$

The function $G$ is written $G(z)=g(z) h(z)$. It satisfies that $G(0)=0$ and, for $z$ small enough, $G$ is increasing. Indeed, from $h(0)=y_{0} / D>0$ we deduce that $h(x)>0$ for $x$ small enough, and from $g(0)=0$ we deduce that $g(x)$ is small for $x$ small enough, so that

$$
G^{\prime}(z)=g^{\prime}(z) h(z)+g(z) h^{\prime}(z) \approx g^{\prime}(z) h(z)>0 .
$$

For $z$ small enough

$$
\frac{1}{G(z)}=\frac{D-\mu g(z)}{g(z)\left(y_{0}-\varphi(z)\right)} \sim \frac{D}{y_{0} g(z)} .
$$

Therefore, using (H3), $\int_{\varepsilon}^{\alpha} \frac{d z}{G(z)}$ converges as $\varepsilon \rightarrow 0$. Using Theorem B.1 the initial value problem (B.3) has a positive nontrivial solution $x_{1}(u)$ such that $x_{1}(u)$ is not identically 0 .

Let us prove now that the initial value problem (B.3) has a solution $\xi\left(u, y_{0}\right)$ satisfying the following conditions:

$$
\xi\left(u, y_{0}\right)>0 \text {, for } 0<u<u_{0} \text { and } \xi\left(u, y_{0}\right)=0 \text {, for } u \leq 0 .
$$

The set $\Phi\left(y_{0}\right)$ of all solutions $u \mapsto x(u)$ of (B.3) defined on a open interval $I$ containing 0 contains at least two distinct solutions, the trivial solution $x_{0}$ for which $x_{0}(u)$ is identically 0 and a non trivial solution $x_{1}$ for which $x_{1}(u)$ is not identically 0 . For each $u \in I$ let

$$
\xi\left(u, y_{0}\right)=\sup _{x \in \Phi\left(y_{0}\right)} x(u) .
$$

It is well known (see [3], section 10.5, problem 7) that $\xi$ belongs to $\Phi\left(y_{0}\right)$. It is called the maximal solution of (B.3) on $I$, corresponding to the initial condition $x(0)=0$. Let $u_{0} \in I$, such that $u_{0}>0$. Using the definition of $\xi$, we have $\xi\left(u, y_{0}\right)>0$ for $0<u<u_{0}$. The trivial solution $x_{0}(u)=0$ of (B.3) corresponds to the trivial solution $\left(x(t)=0, y(0)=y_{0} e^{-D t}\right)$ of $(2.2)$ and a nontrivial solution $x_{1}(u)$ of (B.3) corresponds to a nontrivial positive solution $\left(x_{1}(t), y_{1}(t)\right)$ of $(2.2)$. For instance, to the maximal nontrivial solution $\xi\left(u, y_{0}\right)$ corresponds the maximal solution $\left(X_{1}\left(t, y_{0}\right), Y_{1}\left(t, y_{0}\right)\right)$ of $(2.2)$ constructed as follows. First we consider the second equation in (B.2), where $x$ is replaced by $\xi\left(u, y_{0}\right)$ :

$$
\dot{u}=\mu g\left(\xi\left(u, y_{0}\right)\right)-D, \quad u(0)=0 .
$$


Let $U\left(t, y_{0}\right)$ be the solution of this initial value problem. Since $\xi\left(u, y_{0}\right)=0$ for $u \leq 0$, one has $U\left(t, y_{0}\right)=-D t$ for $u \leq 0$. Using the change of variables $y=y_{0} e^{u}$, the solution $\left(X_{1}\left(t, y_{0}\right), Y_{1}\left(t, y_{0}\right)\right)$ of $(2.2)$ is defined by

$$
X_{1}\left(t, y_{0}\right)=\xi\left(U\left(t, y_{0}\right), y_{0}\right), \quad Y_{1}\left(t, y_{0}\right)=y_{0} e^{U\left(t, y_{0}\right)} .
$$

This solution satisfies $X_{1}\left(t, y_{0}\right)=0, Y_{1}\left(t, y_{0}\right)=y_{0} e^{-D t}$ for $t \leq 0$ and $X_{1}\left(t, y_{0}\right)>0$, $Y_{1}\left(t, y_{0}\right)>0$ for $t<0$, which are the conditions in the theorem.

\section{ACKNOWLEDGMENTS}

The authors would like to thank one anonymous reviewer for interesting comments and suggestions that improved the content of this work. We thank the CNRS-PICS project CODYSYS 278552 and the Euro-Mediterranean research network TREASURE (http://www.inra.fr/treasure) for financial support.

\section{REFERENCES}

1. R. P. Agarwal and V. Lakshmikantham, Uniqueness and non uniqueness criteria for Ordinary Differential Equations, Series in Real Analysis Volume 6, World Scientific 1993.

2. N. Beroual and A. Bendjeddou, On a Predator-Prey System with Holling Functional Response: $\frac{x^{p}}{a+x^{p}}$, Natl. Acad. Sci. Lett. 39 (2016), 43-46. MR34351609

3. J. Dieudonné, Foundations of modern analysis, Academic Press, New York, 1960.

4. H. I. Freedman, Deterministic Mathematical Models in Population Ecology, 2nd ed., HIFR Consulting LTD, Edmonton, Canada, 1987.

5. C.S. Holling, The functional response of predators to prey density and its role in mimicry and population regulation, Mem. Ent. Soc. Can., 45 (1965), 5-60.

6. X.C. Huang and S. J. Merrill, Conditions for uniqueness of limit cycles in general predator-prey systems, Math. Biosci., 96 (1989), 47-60. MR1013301 (90m:92056)

7. Y. Kuang, Global stability of Gause-type predator-prey systems, J. Math. Biol., 28 (1990), 463-474. MR1057049 (91g:92017)

8. Y. Kuang and H. I. Freedman, Uniqueness of limit cycles in Gause-type models of predator-prey systems, Math. Biosci., 88 (1988), 67-84. MR0930003 (89g:92045)

9. Y. Liu, Geometric criteria for the nonexistence of cycles in Gause-type predator-prey systems. Proc. Amer. Math. Soc. 133 (2005), 3619-3626. MR2163598 (2006f:92028)

10. H.L. Smith and P. Waltman, The Theory of the Chemostat: Dynamics of Microbial Competition, vol. 13, Cambridge University Press, Cambridge, 1995.

11. J. Sugie Uniqueness of limit cycles in a predator-prey system with Holling-type functional response, Quart. Appl. Math. 58 (2000), 577--590. MR1770656

12. J. Sugie, M. Katayama, Global asymptotic stability of a predator-prey system of Holling type, Nonlinear Anal. 38 (1999), 105--121. MR1693000

13. J. Sugie, R. Kohno, R. Miyazaki, On a predator-prey system of Holling type, Proc. Amer. Math. Soc. 125 (1997), 2041-2050. MR1396998 (97m:92005)

14. J. Sugie, K. Miyamoto and K. Morino, Absence of limit cycles of a predator-prey system with a sigmoid functional response, Appl. Math. Lett. 9 (1996), 85-90. MR1415457

15. D. Xiao and Z. Zhang, On the uniqueness and nonexistence of limit cycles for predator-prey systems, Nonlinearity, 16 (2003), 1185-1201. MR1975802 (2004c:34084)

Department of Mathematics, University Ferhat Abbes, Sétif, Algeria

E-mail address: n.beroual@univ-setif.dz

itap, Univ Montpellier, Irstea, Montpellier SupAgro, Montpellier, France

E-mail address: tewfik.sari@irstea.fr 